\title{
MRI Signal Computing by Integral and Finite Element Methods
}

\author{
Z. Abidi, B. Bandelier, Member, IEEE, M. Djennah, Member, IEEE
}

\begin{abstract}
Our work models the reception antenna used in magnetic resonance in order to improve the quality of image. When using MRI, several parameters contribute to the image quality. We here want to find the geometrical and electromagnetic characteristics of the antennas which permit to have a signal to noise ratio as great as possible. In our computation, we have taken into account leading factors such as the distance between the probe and the organ to be explored and also the geometrical and electromagnetic characteristics of the probe. A finite element method in the volume and a boundary integral method on its surface are coupled to model the system.
\end{abstract}

Keywords: MRI antennas, boundary integral method, finite element method

\section{INTRODUCTION}

$\mathrm{N}$ OWADAYS, a magnetic resonance imaging is a very interesting exploration tool. Its utility on a patient is such as it gives a spectacular development since many years. However, detection by nuclear magnetic resonance poses a critical problem of sensitivity because the received signal is accompanied by interfering signals and noises of various origins. The interaction between the reception antenna and the human body utilizes several parameters which have a direct relationship with the quality of the image, namely the intrinsic parameters of the molecular structure of human tissue $\left(\rho, \chi, \gamma, \mathrm{T}_{1}, \mathrm{~T}_{2}, ..\right)$. And so the scientist bases his calculations for the design of the antennas

\section{THE PHYSICAL MODEL}

The physical system is made of the organ to explore and of the antenna closed enough to the body surface. The whole is in a MRI environment: a static magnetic field (B0), a system giving field gradients on the three directions of space $3 \mathrm{D}$ and a magnetic radio frequency field (B1). The useful signal which is used to obtain the image comes from the induced body magnetization $\mathrm{M}$ at the time when this one returns to equilibrium with a free precession movement of pulsation $\omega$ and decreasing time T2, as soon as the high frequency field is extinguished; it then produces into the antenna an HF electromagnetic field $\left(e^{\mathrm{s}}, h^{\mathrm{s}}\right)$. We study two different models of antennas. The first one is composed with metallic material, the second one is a thin conducting layer impregnated on a dielectric substrate.

Zakia Abidi is with the U2R2M, University of Paris XI, IEF Bât 220, 91405 Orsay France (phone 33-169157978; fax 33-1-69154136; e-mail: zakia.abidi@u-psud.fr)

Bernard Bandelier is with the U2R2M, University of Paris XI, IEF Bât 220, 91405 Orsay France (phone 33-169157978; fax 33-169154136; e-mail: bernard.bandelier@u-psud.fr)

Mohammed Djennah is with National Polytechnic School Algiers,Algeria BP 03 Bis Ben Aknoun 16033 Algiers (phone 213661120002 ;fax: 213-21522973; e-mail: zakimoza@yahoo.com)
Both of the antennas are closed enough to the body to explore, in order to have a maximum intensity of the magnetic flux created by the induced magnetisation of the organ.

The distance between the body and the antenna is a determining factor in the design of this one, a very weak distance would cause the increase in the intensity of the noise which results in two physical phenomenon: the capacitive effect and the inductive effect between the body and the antenna; a more or less large distance giving a loss of the signal. This factor does not directly appear in the equation which governs our physical system; it intervenes like a variable parameter in the calculation of the field's source. The last is written in the form:

$$
\left(e^{s}, h^{s}\right)=\left(E^{s}, H^{s}\right) e^{-\left(i \omega+\frac{1}{T 2}\right) t}
$$

$\omega=2 \pi f$, where $f$ is frequency of magnetic field (B1) which must be fixed at the resonance frequency of targeted nucleus of the body to explore and T2, is a transverse relaxation time.

The system thus defined is governed by Maxwell's equations:

$$
\left\{\begin{array}{l}
\operatorname{curl} h-(\sigma-\propto \varepsilon) e=0 \\
\text { curl } e-\propto \mu h=0
\end{array}\right.
$$

Where $\propto=\left(i \omega+1 / T_{2}\right)$ and $\mu, \quad \sigma, \quad \varepsilon \quad$ are the electromagnetic characteristics of the material.

\section{FORMULATION OF THE PROBLEM}

We adopt a variational formulation with magnetic and electric fields. We consider $\Omega$ as the domain of the antenna in space $R^{3}$ with conductivity $\sigma$, permeability $\mu$ and permittivity $\varepsilon$; its surface is $\Gamma$ and $n$ is the normal vector to $\Gamma$. In the first case, where the antenna is a metallic domain, the variational formulation is made of a volume integral and a surface integral. In second case, where antenna is impregnated on a dielectric substrate, it is simulated as surface and variational formulation is composed of only surface integral.

\section{A. Formulation on Domain of Antenna :}

By respectively multiplying (1) and (2) by test functions (e') and (curl e') and by integrating on domain $\Omega$ we obtain:

$$
\begin{aligned}
& \int_{\Omega} \operatorname{curl} h . e^{\prime}-(\sigma-\varepsilon \alpha) \int_{\Omega} \text { e. } e^{\prime}=0 \\
& \int_{\Omega} \text { h.curl } e^{\prime}=\frac{1}{\alpha \mu} \int_{\Omega} \text { curl e.curl } e^{\prime}
\end{aligned}
$$

The first integral of the first term can be written as: 


$$
\begin{aligned}
\int_{\Omega} \operatorname{curl} h \cdot e^{\prime}= & \int_{\Omega} h \cdot \operatorname{curl} e^{\prime}+\int_{\Gamma}(n \wedge e) \cdot h^{\prime} \\
& \frac{1}{\alpha \mu} \int_{\Omega} \operatorname{curl} e \cdot \operatorname{curl} e^{\prime} \\
& -(\sigma-\alpha \varepsilon) \int_{\Omega} e \cdot e^{\prime} \\
& -\int_{\Gamma}(h \Lambda \mathrm{n}) \cdot e^{\prime}=0
\end{aligned}
$$

Also, by multiplying (1) and (2) by test functions (rot h') and (h') respectively and integrating on domain $\Omega$ we obtain:

$$
\begin{aligned}
& \int_{\Omega} \text { curl h.curl } h^{\prime}-(\sigma-\varepsilon \alpha) \int_{\Omega} \text { e.curl } h^{\prime}=0 \\
& \int_{\Omega} h \cdot h^{\prime}=\frac{1}{\alpha \mu} \int_{\Omega} \text { curle. } h^{\prime}
\end{aligned}
$$

The last term can also be written:

$$
\int_{\Omega} \operatorname{curl} e \cdot h^{\prime}=\int_{\Omega} e \cdot \operatorname{curl} h^{\prime}-\int_{\Gamma}(n \wedge e) \cdot h^{\prime}
$$

And finally we obtain:

$$
\begin{gathered}
\frac{1}{(\sigma-\alpha \varepsilon)} \int_{\Omega} \text { curl h.curl } h^{\prime}-\mu \alpha \int_{\Omega} h \cdot h^{\prime} \\
-\int_{\Gamma}(e \Lambda \mathrm{n}) \cdot h^{\prime}=0
\end{gathered}
$$

\section{B. Formulation of the Boundary Term:}

Our problem is defined in all space $\mathrm{R}^{3}$, the exterior problem (air) is considered by a formulation of boundary integral. We can write the tangential component of electromagnetic field by integral representation where the potential define the jump of the electromagnetic field.

The Stratton-Chu formulae [4] give an integral representation of the electromagnetic fields. Outside $\Omega$, for $x \notin \Gamma$, we have:

$$
\begin{gathered}
e(x)=-\frac{1}{\varepsilon_{0} \alpha} \operatorname{grad}_{\Gamma} \int_{\Gamma} G(x, y) \operatorname{div}(n \Lambda h(y)) \\
+\alpha \mu_{0} \int_{\Gamma} \mathrm{G}(\mathrm{x}, \mathrm{y})(\mathrm{n} \Lambda \mathrm{h}(\mathrm{y}) \\
\quad+\operatorname{curl} \int_{\Gamma} \mathrm{G}(\mathrm{x}, \mathrm{y})(\mathrm{n} \Lambda \mathrm{e}(\mathrm{y})) \\
h(x)=\frac{1}{\mu_{0} \alpha} \operatorname{grad}_{\Gamma} \int_{\Gamma} G(x, y) \operatorname{div}(n \Lambda e(y)) \\
\quad-\alpha \varepsilon_{0} \int_{\Gamma} \mathrm{G}(\mathrm{x}, \mathrm{y})(\mathrm{n} \Lambda \mathrm{e}(\mathrm{y})) \\
\quad+\operatorname{curl} \int_{\Gamma} \mathrm{G}(\mathrm{x}, \mathrm{y}) \Lambda(\mathrm{n} \Lambda \mathrm{h}(\mathrm{y}))
\end{gathered}
$$

where, $G(x, y)=e^{-i k\|x-y\|} / 4 \pi\|x-y\|$ is the Green function in the air for the Helmoltz equation and $\mathrm{k}$ is the wave number.

By taking into account that:

$$
\begin{gathered}
\lim _{x \rightarrow x 0} n\left(x_{0}\right) \Lambda \operatorname{curl} \int_{\Gamma} \mathrm{G}(\mathrm{x}, \mathrm{y}) \mathrm{u}(\mathrm{y})= \\
\frac{u_{0}(x)}{2}+n\left(x_{0}\right) \Lambda \int_{\Gamma} \operatorname{grad}_{x} G\left(x_{0}, y\right) \Lambda u(y)
\end{gathered}
$$

we obtain the tangential trace of the electromagnetic fields $h$ and e on the external face of $\Gamma$

$$
\begin{gathered}
\frac{n \Lambda \mathrm{h}(\mathrm{x})}{2}=n \Lambda h^{s}(x) \\
+\frac{1}{\mu_{0} \alpha} n \Lambda \operatorname{grad}_{\Gamma} \int_{\Gamma} G(x, y) \operatorname{div}(n \Lambda e(y)) \\
-\alpha \varepsilon_{0}\left(n \Lambda \int_{\Gamma} \mathrm{G}(\mathrm{x}, \mathrm{y})(\mathrm{n} \Lambda \mathrm{e}(\mathrm{y}))\right. \\
+\mathrm{n} \Lambda \int_{\Gamma} \operatorname{grad}_{\mathrm{x}} \mathrm{G}(\mathrm{x}, \mathrm{y}) \Lambda(\mathrm{n} \Lambda \mathrm{h}(\mathrm{y})) \\
\frac{n \Lambda \mathrm{e}(\mathrm{x})}{2}=n \Lambda e^{s}(x) \\
+\frac{1}{\varepsilon_{0} \alpha} n \Lambda \operatorname{grad}_{\Gamma} \int_{\Gamma} G(x, y \operatorname{div}(n \Lambda h(y)) \\
-\alpha \mu_{0} n \Lambda \int_{\Gamma} \mathrm{G}(\mathrm{x}, \mathrm{y})(\mathrm{n} \Lambda \mathrm{h}(\mathrm{y}) \\
+\mathrm{n} \Lambda \int_{\Gamma} \operatorname{grad}_{\mathrm{x}} \mathrm{G}(\mathrm{x}, \mathrm{y}) \Lambda(\mathrm{n} \Lambda \mathrm{e}(\mathrm{y}))
\end{gathered}
$$

\section{Variational Formulation of Boundary Term:}

By multiplying (13) by a tangential test field (e') defines in the same functional space of electric field and by integrating on $\Gamma$, we obtain:

$$
\begin{aligned}
& \int_{\Gamma}(n \Lambda \mathrm{h}(\mathrm{x})) \mathrm{e}^{\prime}(\mathrm{x})=2 \int_{\Gamma}\left(n \Lambda h^{s}(x)\right) e^{\prime}(x) \\
& +2 \alpha \varepsilon_{0} \int_{\Gamma} \int_{\Gamma} \mathrm{G}(\mathrm{x}, \mathrm{y})(\mathrm{n} \Lambda \mathrm{e}(\mathrm{x}))\left(\mathrm{n} \Lambda \mathrm{e}^{\prime}(\mathrm{x})\right) \\
& -2 \int_{\Gamma} \int_{\Gamma} \operatorname{grad} \mathrm{G}(\mathrm{x}, \mathrm{y}) \Lambda(\mathrm{n} \Lambda \mathrm{h}(\mathrm{x})) \cdot\left(\mathrm{n} \Lambda \mathrm{e}^{\prime}(\mathrm{x})\right) \\
& +\frac{2}{\mu_{0} \alpha} \int_{\Gamma} \int_{\Gamma} G(x, y) \operatorname{div}\left(n \Lambda \mathrm{e}(\mathrm{x}) \cdot \operatorname{div}\left(n \Lambda \mathrm{e}^{\prime}(\mathrm{x})\right)\right.
\end{aligned}
$$

In the same way, we multiply (14) by tangential test field (h') defined in the same functional space of magnetic field and integrate on $\Gamma$ and then we obtain:

$$
\begin{aligned}
& \int_{\Gamma}(n \Lambda \mathrm{e}(\mathrm{x})) \mathrm{h}^{\prime}(\mathrm{x})=2 \int_{\Gamma}\left(n \Lambda e^{s}(x)\right) h^{\prime}(x) \\
& -\frac{2}{\varepsilon_{0} \alpha} \int_{\Gamma} \int_{\Gamma} G(x, y) \operatorname{div}(n \Lambda h(x)) \cdot \operatorname{div}\left(n \Lambda \mathrm{h}^{\prime}(\mathrm{x})\right) \\
& -2 \alpha \mu_{0} \int_{\Gamma} \int_{\Gamma} \mathrm{G}(\mathrm{x}, \mathrm{y})(\mathrm{n} \Lambda \mathrm{h}(\mathrm{x}))\left(\mathrm{n} \Lambda \mathrm{h}^{\prime}(\mathrm{x})\right) \\
& -2 \int_{\Gamma} \int_{\Gamma} \operatorname{gradx} \mathrm{G}(\mathrm{x}, \mathrm{y}) \Lambda(\mathrm{n} \Lambda \mathrm{e}(\mathrm{x})) \cdot\left(\mathrm{n} \Lambda \mathrm{h}^{\prime}(\mathrm{x})\right)
\end{aligned}
$$


Finally, by replacing the boundary terms (15), (16) by their expressions on (6) and (10), we obtain the global variational formulation with the two electromagnetic fields (e,h):

$$
\begin{gathered}
\quad \frac{1}{\alpha \mu} \int_{\Omega} \text { curl e.curl } e^{\prime}-(\sigma-\alpha \varepsilon) \int_{\Omega} e \cdot e^{\prime} \\
+\frac{2}{\mu_{0} \alpha} \int_{\Gamma} \int_{\Gamma} G(x, y) \operatorname{div}\left(n \Lambda \mathrm{e}(\mathrm{x}) \cdot \operatorname{div}\left(n \Lambda \mathrm{e}^{\prime}(\mathrm{x})\right)\right. \\
+2 \alpha \varepsilon_{0} \int_{\Gamma} \int_{\Gamma} \mathrm{G}(\mathrm{x}, \mathrm{y})(\mathrm{n} \Lambda \mathrm{e}(\mathrm{x}))\left(\mathrm{n} \Lambda \mathrm{e}^{\prime}(\mathrm{x})\right) \\
-2 \int_{\Gamma} \int_{\Gamma} \operatorname{grad} \mathrm{G}(\mathrm{x}, \mathrm{y}) \Lambda(\mathrm{n} \Lambda \mathrm{h}(\mathrm{x})) \cdot\left(\mathrm{n} \Lambda \mathrm{e}^{\prime}(\mathrm{x})\right) \\
=-2 \int_{\Gamma}\left(n \Lambda h^{s}(x)\right) e^{\prime}(x) \\
-\frac{1}{(\sigma-\alpha \varepsilon)} \int_{\Omega} \operatorname{curl} h \cdot \operatorname{curl} h^{\prime}-\mu \propto \int_{\Omega} h \cdot h^{\prime} \\
-2 \alpha \mu_{0} \int_{\Gamma} \int_{\Gamma} G(x, y) \operatorname{div}(n \Lambda h(x)) \cdot \operatorname{div}\left(n \Lambda \mathrm{h}^{\prime}(\mathrm{x})\right) \\
-2 \int_{\Gamma} \int_{\Gamma} \operatorname{gradx} \mathrm{G}(\mathrm{x}, \mathrm{y}) \Lambda(\mathrm{n} \Lambda \mathrm{e}(\mathrm{x})) \cdot\left(\mathrm{n} \Lambda \mathrm{h}^{\prime}(\mathrm{x})\right) \\
\left.=2 \int_{\Gamma}\left(n \Lambda e^{s}(x)\right) h^{\prime}(x)\right)\left(\mathrm{n} \Lambda \mathrm{h}^{\prime}(\mathrm{x})\right)
\end{gathered}
$$

\section{COMPUTATION OF THE SOURCE FIELDS}

The works which was realized by [2]-[7] on the same antenna model aims to find resonance frequency of this one as a function of its geometrical characteristics. But our work aims to find a configuration of the system defined above which gives a signal to noise ratio as great as possible. We have used a real physical data of an MRI sequence, such: a static magnetic field $\mathrm{B}_{0}=1.5 \mathrm{~T}$, a radio frequency magnetic field $B_{1}=B_{01} e^{-i \omega 0 t}$, where $\omega_{0}$ is defined in [6].

The median magnetization of body:

$M=M_{0} e^{-i \omega 0 t}=M_{z}(T 1) \cos (\theta)+M_{x y}(T 2) \sin (\theta)$, $\theta=\gamma B_{01} \tau$.

$\tau$ is the application time of $B_{1}$

$\mathrm{B}_{01}$ is the amplitude of field $\mathrm{B}_{1}$

The tangential component $M_{x y}$ represents the source field of the image signal. Then, we can write our source field under form:

$$
\left\{\begin{array}{c}
e^{s}(x, t)=\frac{\mu_{0} \alpha}{4 \pi} \int_{\Gamma_{-} \text {body }} \frac{M \wedge n}{r} \\
h^{s}(s, t)=\frac{1}{4 \pi} \int_{\Gamma_{-} \text {body }} \frac{(M \wedge n) \wedge r}{r^{3}}
\end{array}\right.
$$

and $\mathrm{M}$ is defined by:

$$
M\left(\begin{array}{c}
M_{x} \\
M_{y} \\
M_{z}
\end{array}\right) ;\left\{\begin{array}{c}
M_{x}=M_{0} \cos (\theta) \sin (x) \\
M_{y}=M_{0} \cos (\theta) \cos (x) \\
M_{z}=0
\end{array}\right.
$$

where $\varphi=\alpha * \frac{I T P}{T_{2}} ; I T P=1, N S T E P$

NSTEP is the number of iterations in time domain.

\section{V- NUMERICAL RESOLUTION}

To solve the variational problem defined by both of the equations (17) and (18), we adopt the finite element method, where we took the circulation of the fields $\mathrm{E}$ and $\mathrm{H}$ along the edges of the grid as the unknowns of the problem.

When the antenna is a metallic layer impregnated on a dielectric substrate, it is considered as a set of triangle on $\Gamma$ of conductivity $\sigma_{\mathrm{c}}$ multiplied by the thickness of the layer.

For the discretization we used Whitney elements [5]. Each of the two fields e and $\mathrm{h}$ is written in the form according to the following form:

$$
u=\sum_{I E=1}^{N B E} U_{i j} w_{i j}
$$

where:

NBE: is the number of the edges

IE: describes the edges of the elements of the grid

$\mathrm{U}_{\mathrm{ij}}$ : is the circulation of the field on the edge of top ij

$$
w_{i j}=\lambda_{i} \nabla \lambda_{j}-\lambda_{j} \nabla \lambda_{i}
$$

$\lambda_{i}$ : barycentric coordinate of the element of the grid We replace each of the fields $(e, h)$ by their discrete form

$$
h=\sum_{I E=1}^{N B E} h_{i j} w_{i j} \quad, \quad e=\sum_{I E=1}^{N B E} e_{i j} w_{i j}
$$

The global variational formulation is written in the following matrix form:

$$
\text { A. } F=B
$$

where $\mathrm{A}$ is a square matrix, constituted with the geometrical characteristics of the grid and the electromagnetic characteristics of the materials $\mathrm{F}=(\mathrm{h}, \mathrm{e})$ is the unknowns vector and $\mathrm{B}$ is the sources vector.

\section{NUMERICAL RESULTS}

We used only two models of antennas with different geometrical forms. We simulated the body with a sphere with a median induced magnetization M0. By varying the distance between the antenna and the sphere, we traced the arrows of the electromagnetic field solution of the problem.

Figures bellow show the structures of grid used: 


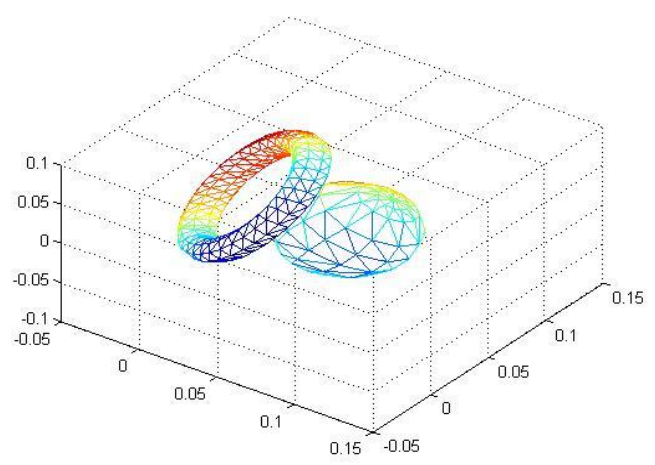

Fig. 1. Antenna-Body grid

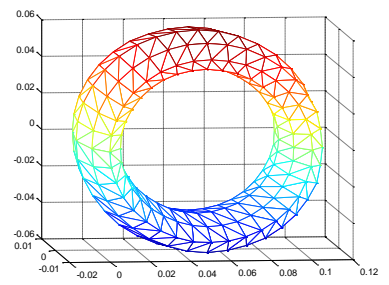

Fig. 2. Torus antenna grid

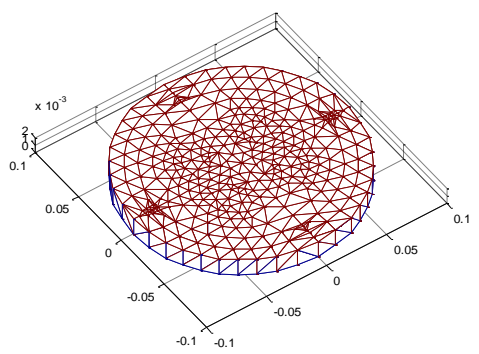

Fig. 3. Metallic layer on dielectric substrate (circular form)

The following figures show the distribution of the signal induced by a transverse magnetization $\mathrm{M}$ of the body.

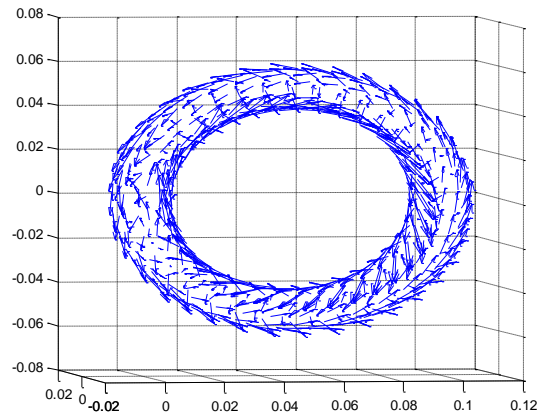

Fig. 4. Arrows of electrical field on Torus

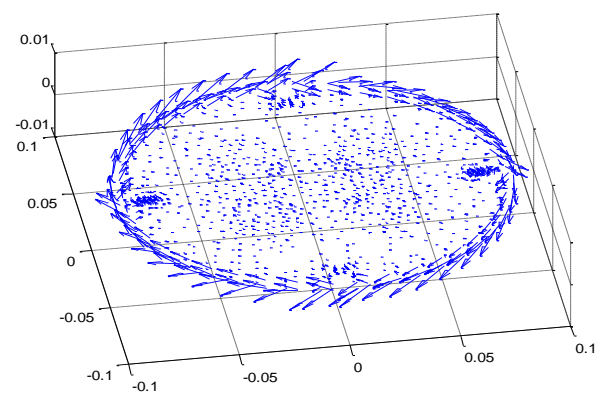

Fig. 5. Distribution of the signal induced in impregnated antenna

\section{VI- CONCLUSION}

In this paper, we present preliminary numerical results of our computation code. From these results, we deduce that the intensity of the electromagnetic flux created by the induced magnetization of the organ and embraced by the surface antenna is very sensible to variation of the distance between the antenna and the body to explore, and to their geometrical forms. Thus, we remark that for a spherical form of the body to explore the torus antenna is more adopted than a square form of the antenna.

From ten to one hundred Mhz, [the bandwidth of resonance frequency of biological tissue] the induced signal is globally supported by the surface $\Gamma$ of the antenna that is why a Stratton-Chu integral formula is adopted in our computation.

The induced signal is characterized by a very low intensity and a very fast attenuation. This requires a particular numerical processing. The 'pseudo-period' of $\mathrm{M}_{\mathrm{xy}}$ is $\mathrm{T}=2 \pi / \gamma \mathrm{B} 0=3.10^{-7}$ second for protons subjected to a field of 0.5 Tesla. Our work represents a new approach in this field.

Our computation code doesn't converge when the distance between the antenna and the organ is less than $0.01 \mathrm{~cm}$ for antenna about $5 \mathrm{~cm}$ of median radius.

By taken away the antenna from the organ, we loose in the signal intensity but we gain CPU time and the convergence of the computation code.

In our computation, we don't take into account physical phenomena, such us, inductive and capacitive effects between antenna and the organ. These effects accentuate when the distance is towards zero. These effects essentially contribute in the identification of noise and can be quantify by experimental measurements.

\section{REFERENCES}

[1] M. Djennah, F. D. Rioux, B. Bandelier "Computation of electric charges and eddy currents with an e formulation" IEEE Transaction Magnetic 1997, Vol 32, pp 1322-25

[2] B.Kebaili, B.Bandelier,and F.Rioux-Damidau,"Modelling a thin shell system submitted to an electromagnetic wave", IEEE Trans.Mag., vol 37, 2001, pp.3277-3280

[3] D.C. Dibben and A. C. Metaxas, "Finite element time domain analysis of multimode applicators using edge elements" J. Microwave, power, Electromagn. Energy. Vol 29, n 4, 44. 242-250, 1994.

[4] J.A Stratton, Electromagnetic theory, Mac Graw Hill,New York, 1941

[5] J.C.Nédélec,"Mixed Finite Elements in R3", Numer.Math.,vol.35, 1980,pp.315-341

[6] A.Alaux,"l'Image par Resonance Magnétique"SAURAMPS MEDICAL, 1994

[7] L.Darrasse, J-C Jinefri "Perspectives with cryogenic RF robes in biomedical MRI’ELSEVIER BIOCHIMI 2003, Vol 85,pp915-937 